\title{
Weak solutions nonlinear fractional integrodifferential equations in nonreflexive Banach spaces
}

\section{Baolin Li $i^{*}$ and Haide Gou}

\section{"Correspondence:} ghdzxh@163.com

College of Mathematics and Statistics, Northwest Normal University, Lanzhou, 730070, P.R. China

\section{Springer}

\begin{abstract}
The aim of this paper is to discuss the existence of weak solutions for a nonlinear two-point boundary value problem of integrodifferential equations of fractional order $\alpha \in(1,2]$. Our analysis relies on the Krasnoselskii fixed point theorem combined with the technique of measure of weak noncompactness.
\end{abstract}

MSC: 26A39; 35D30; 45B05; 35S15

Keywords: weak solutions; Henstock-Kurzweil-Pettis integral; Caputo fractional derivative; measure of weak noncompactness; boundary value problem

\section{Introduction}

Fractional differential equations arise in many engineering and scientific disciplines as the mathematical modeling of systems and processes in the fields of physics, chemistry, aerodynamics, electrodynamics of complex medium, polymer rheology, and they have been emerging as an important area of investigation in the last few decades; see [1-8]. However, the theory of boundary value problems for nonlinear fractional differential equations is still in the initial stages and many aspects of this theory need to be explored.

In [9], Zhou discusses the existence of solutions for a nonlinear multi-point boundary value problem of integrodifferential equations of fractional order as follows:

$$
\left\{\begin{array}{l}
{ }^{c} D_{0+}^{\alpha} x(t)=f(t, x(t),(H x)(t),(K x)(t)), \quad t \in[0,1], \alpha \in(1,2], \\
a_{1} x(0)-b_{1} x^{\prime}(0)=d_{1} x\left(\xi_{1}\right), \quad a_{2} x(1)+b_{2} x^{\prime}(1)=d_{2} x\left(\xi_{2}\right),
\end{array}\right.
$$

where ${ }^{c} D_{0_{+}}^{\alpha}$ denotes the fractional Caputo derivative and

$$
(H x)(s)=\int_{0}^{t} g(t, s) u(s) d s, \quad(K x)(s)=\int_{0}^{t} h(t, s) d s,
$$

with respect to the strong topology. In [10], Bouffak investigates the existence of weak solutions for a class of boundary value problem of fractional differential equations involving nonlinear integral conditions of the form

$$
\left\{\begin{array}{l}
{ }^{c} D_{0+}^{\alpha} x(t)=f(t, x(t)), \quad t \in[0, T], \alpha \in(0,1], \\
x(0)+\mu \int_{0}^{T} x(s) d s=x(T),
\end{array}\right.
$$

(c) The Author(s) 2016. This article is distributed under the terms of the Creative Commons Attribution 4.0 International License (http://creativecommons.org/licenses/by/4.0/), which permits unrestricted use, distribution, and reproduction in any medium, provided you give appropriate credit to the original author(s) and the source, provide a link to the Creative Commons license, and indicate if changes were made. 
by used of the measure of weak noncompactness and Pettis integrals.

In recent years, the theory for boundary value problem of integrodifferential equations of fractional order in Banach spaces endowed with its weak topology has been few studied until now and, in [11], Li and Gou discussed the existence theorem of weak solutions for a class of nonlinear integral equations and obtain a new results by using the techniques of measure of weak noncompactness and Henstock-Kurzweil-Pettis integrals, motivated by this work, in this paper, we use the techniques of the measure of weak noncompactness combined with the fixed point theorem to discuss the existence theorem of weak solutions for a class of nonlinear fractional integrodifferential equations of the form

$$
\left\{\begin{array}{l}
{ }^{c} D_{0+}^{\alpha} x(t)=f(t, x(t),(T x)(t),(S x)(t)), \quad t \in[0,1], \alpha \in(1,2], \\
a_{1} x(0)-a_{2} x^{\prime}(0)=\gamma_{1}, \quad b_{1} x(1)+b_{2} x^{\prime}(1)=\gamma_{2},
\end{array}\right.
$$

where ${ }^{c} D_{0+}^{\alpha}$ denotes the fractional Caputo derivative and

$$
(T x)(s)=\int_{0}^{s} k_{1}(s, \tau) g(\tau, x(\tau)) d \tau, \quad(S x)(s)=\int_{0}^{1} k_{2}(s, \tau) h(\tau, x(\tau)) d \tau,
$$

$f: I \times E^{3} \rightarrow E$ is a given function satisfying some assumptions that will be specified later, $E$ is a nonreflexive Banach space and the integrals are taken in the sense of HenstockKurzweil-Pettis. Also, it is assumed that $a_{i}, b_{i} \geq 0, \gamma_{i}, i=1,2$ are real numbers.

The paper is organized as follows: In Section 2 we recall some basic known results. In Section 3 we discuss the existence theorem of weak solutions for the problem (1.1).

\section{Preliminaries}

In this section, we introduce notations, definitions, and preliminary results which will be used throughout this paper.

Let $I=[0,1]$ be the real interval, let $E$ be a real Banach space with norm $\|\cdot\|$ and its dual space $E^{*}$, and also $E_{w}=(E, w)=\left(E, \sigma\left(E, E^{*}\right)\right)$ denotes the space $E$ with its weak topology. Denote by $C\left(I, E_{\omega}\right)=(C(I, E), \omega)$ the space of all continuous functions from $I$ to $E$ endowed with the weak topology and the usual supremum norm $\|x\|_{\infty}=\sup _{t \in I}\|x(t)\|$.

The fundamental tool in this paper is the measure of weak noncompactness developed by De Blasi, for more details see [12].

Now, for the convenience of the reader, we recall some useful definitions of integrals.

Definition 2.1 ([13]) A function $u: I \rightarrow E$ is said to be Henstock-Kurzweil integrable on $I$ if there exists an $J \in E$ such that, for every $\varepsilon>0$, there exists $\delta(\xi): I \rightarrow \mathbb{R}^{+}$such that, for every $\delta$-fine partition $D=\left\{\left(I_{i}, \xi_{i}\right)\right\}_{i=1}^{n}$, we have

$$
\left\|\sum_{i=1}^{n} u\left(\xi_{i}\right) \mu\left(I_{i}\right)-J\right\|<\varepsilon
$$

we denote the Henstock-Kurzweil integral $J$ by $(H K) \int_{a}^{b} u(s) d s$.

Definition 2.2 ([13]) A function $f: I \rightarrow E$ is said to be Henstock-Kurzweil-Pettis integrable, or simply HKP-integrable on $I$, if there exists a function $g: I \rightarrow E$ with the following properties: 
(i) $\forall x^{*} \in E^{*}, x^{*} f$ is Henstock-Kurzweil integrable on $I$;

(ii) $\forall t \in I, \forall x^{*} \in E^{*}, x^{*} g(t)=(H K) \int_{0}^{t} x^{*} f(s) d s$.

This function $g$ will be called a primitive of $f$ and we will denote by $g(t)=\int_{0}^{t} f(t) d t$ the Henstock-Kurzweil-Pettis integral of $f$ on the interval $I$.

Theorem 2.1 ([13]; mean value theorem for the HKP integral) If the function $f: I_{\alpha} \rightarrow E$ is HKP integrable, then

$$
\int_{I} f(t) d t \in|I| \cdot \overline{\operatorname{conv}} f(I)
$$

where $\overline{\operatorname{co}} f(I)$ is the closure of the convex of $f(I)$, I is an arbitrary subinterval of $I_{\alpha}$ and $|I|$ is the length of $I$.

Theorem 2.2 ([14]) Let $f: I \rightarrow E$ and assume that $f_{n}: I \rightarrow E, n \in N$, are HKP integrable on I. For each $n \in N$, let $F_{n}$ be a primitive of $f_{n}$. If we assume that:

(i) $\forall x^{*} \in E^{*}, x^{*}\left(f_{n}(t)\right) \rightarrow x^{*}(f(t))$ a.e. on $I$,

(ii) for each $x^{*} \in E^{*}$, the family $G=\left\{x^{*} F_{n}: n=1,2, \ldots\right\}$ is uniformly $A C G_{*}$ on $I$ (i.e. weakly uniformly $A_{C G_{*}}$ on $I$ ),

(iii) for each $x^{*} \in E^{*}$, the set $G$ is equicontinuous on I, then $f$ is HKP integrable on I and $\int_{0}^{t} f_{n}(s) d s$ tends weakly in $E$ to $\int_{0}^{t} f(s) d s$ for each $t \in I$.

Lemma 2.1 ([15]) If $B \subset C(I, E)$ is equicontinuous, $u_{0} \in C(I, E)$, then $\overline{c o}\left\{B, u_{0}\right\}$ is also equicontinuous in $C(I, E)$.

Lemma 2.2 ([15]) If $B \subset C(I, E)$ is equicontinuous and bounded, then $\beta(B)=$ $\max _{t \in I} \beta(B(t))$.

Lemma 2.3 ([15]) If $B \subset C(I, E)$ is equicontinuous and bounded, then $\beta(B(t)) \in C(I, \mathbb{R}+)$ and

$$
\beta(B(s) d s) \leq \beta(B(s)) d s, \quad \forall t \in I .
$$

We give some fixed point theorem, which play a key role in the proofs of our main results.

Theorem 2.3 ([15]) Let $M$ be a nonempty bounded closed convex subset of a Banach space E. Suppose that $T: M \rightarrow M$ is weakly sequentially continuous and there exist an integer $n_{0}$ and a vector $x_{0} \in M$ such that $T$ is power-convex condensing about $x_{0}$ and $n_{0}$. Then $T$ has at least one fixed point in $M$.

For completeness we recall the definition of the Caputo derivative of fractional order.

Definition 2.3 ([16]) Let $x: I \rightarrow E$ be a function. The fractional HKP-integral of the function $x$ of order $\alpha \in \mathbb{R}_{+}$is defined by

$$
I_{0+}^{\alpha} x(t):=\int_{0}^{t} \frac{(t-s)^{\alpha-1}}{\Gamma(\alpha)} x(s) d s .
$$

In the above definition the sign ' $\int$ ' denotes the HKP-integral. 
Lemma 2.4 For a function $f: I \rightarrow E$, the Caputo fractional order derivative of $f$ is defined by

$$
{ }^{c} D_{0+}^{\alpha} f(t)=\frac{1}{\Gamma(n-\alpha)} \int_{0}^{t}(t-s)^{n-\alpha-1} f^{(n)}(s) d s, \quad n-1<\alpha<n,
$$

where $n=[\alpha]+1$ and $[\alpha]$ denotes the integer part of $\alpha$.

\section{Main results}

In this section, we prove the existence of solutions to the problem (1.1) in the space $C\left(I, E_{\omega}\right)$. Let us start by defining what we mean by a solution of the problem (1.1).

Definition 3.1 A function $x \in C\left(I, E_{w}\right)$ is said to be a solution of the problem (1.1) if $x$ satisfies the equation ${ }^{c} D_{0+}^{\alpha} x(t)=f(t, x(t),(T x)(t),(S x)(t))$ on $I$ and satisfies the conditions $a_{1} x(0)-a_{2} x^{\prime}(0)=\gamma_{1}, b_{1} x(1)+b_{2} x^{\prime}(1)=\gamma_{2}$.

Lemma 3.1 Let $\alpha>0$, then the differential equation

$$
{ }^{c} D_{0+}^{\alpha} u(t)=0
$$

has a solution $u(t)=c_{0}+c_{1} t+c_{2} t^{2}+\cdots+c_{n} t^{n-1}, c_{i} \in \mathbb{R}, i=0,1, \ldots, n, n=[\alpha]+1$.

From the lemma above, we deduce the following statement.

Lemma 3.2 Let $\alpha>0$, then

$$
I_{0+}^{\alpha}\left({ }^{c} D_{0+}^{\alpha} u(t)\right)=u(t)+c_{0}+c_{1} t+c_{2} t^{2}+\cdots+c_{n} t^{n-1}
$$

for some $c_{i} \in \mathbb{R}, i=0,1, \ldots, n, n=[\alpha]+1$.

We derive the corresponding Green's function for the boundary value problem (1.1) which will play major role in our next analysis.

Lemma 3.3 Let $\rho \in C\left(I, E_{w}\right)$ and $\alpha \in(1,2]$, then the unique solution of

$$
\left\{\begin{array}{l}
{ }^{c} D_{0+}^{\alpha} x(t)=\rho(t), \quad t \in I, \\
a_{1} x(0)-a_{2} x^{\prime}(0)=\gamma_{1}, \quad b_{1} x(1)+b_{2} x^{\prime}(1)=\gamma_{2},
\end{array}\right.
$$

is given by

$$
x(t)=\int_{0}^{1} G(t, s) \rho(s) d s+\frac{\left(b_{1}+b_{2}\right) \gamma_{1}+a_{2} \gamma_{2}}{l}+\frac{a_{1} \gamma_{2}-b_{1} \gamma_{1}}{l} t,
$$

where the Green's function $G$ is given by

$$
G(t, s)=\left\{\begin{array}{cc}
\frac{1}{\Gamma(\alpha)}\left[(t-s)^{\alpha-1}-\frac{a_{2} b_{1}}{l}(1-s)^{\alpha-1}-\frac{a_{1} b_{1}}{l}(1-s)^{\alpha-1} t\right] & \\
\quad+\frac{1}{\Gamma(\alpha-1)}\left[-\frac{a_{2} b_{2}}{l}(1-s)^{\alpha-2}-\frac{a_{1} b_{2}}{l}(1-s)^{\alpha-2} t\right], & 0 \leq s \leq t, \\
\frac{1}{\Gamma(\alpha)}\left[-\frac{a_{2} b_{1}}{l}(1-s)^{\alpha-1}-\frac{a_{1} b_{1}}{l}(1-s)^{\alpha-1} t\right] & \\
\quad+\frac{1}{\Gamma(\alpha-1)}\left[-\frac{a_{2} b_{2}}{l}(1-s)^{\alpha-2}-\frac{a_{1} b_{2}}{l}(1-s)^{\alpha-2} t\right], & t \leq s<1,
\end{array}\right.
$$

and $p(t)=\frac{\left(b_{1}+b_{2}\right) \gamma_{1}+a_{2} \gamma_{2}}{l}+\frac{a_{1} \gamma_{2}-b_{1} \gamma_{1}}{l} t, l=a_{1} b_{1}+a_{1} b_{2}+a_{2} b_{1} \neq 0, a_{i}, b_{i} \geq 0, i=1,2$. 
Proof Assume that $x(t)$ satisfies (3.1), then Lemma 3.2 implies that

$$
x(t)=I_{0+}^{\alpha} \rho(t)-c_{1}-c_{2} t=\frac{1}{\Gamma(\alpha)} \int_{0}^{t}(t-s)^{\alpha-1} \rho(t) d s-c_{1}-c_{2} t
$$

for some constants $c_{1}, c_{2} \in \mathbb{R}$.

On the other hand, by the relations $D_{0+}^{\alpha} I_{0+}^{\alpha} x(t)=x(t)$ and $I_{0+}^{\alpha} I_{0+}^{\beta} x(t)=I_{0+}^{\alpha+\beta} x(t)$, for $\alpha, \beta>$ $0, x \in C\left(I, E_{w}\right)$, we have

$$
x^{\prime}(t)=\frac{1}{\Gamma(\alpha-1)} \int_{0}^{t}(t-s)^{\alpha-2} \rho(s) d s-c_{2} .
$$

By the boundary conditions of (3.1), we obtain

$$
\left\{\begin{array}{l}
-a_{1} c_{1}+a_{2} c_{2}=\gamma_{1}-a_{1} I_{0+}^{\alpha} \rho(0)+a_{2} I_{0+}^{\alpha-1} \rho(0), \\
-b_{1} c_{1}-\left(b_{1}+b_{2}\right) c_{2}=\gamma_{2}-b_{2} I_{0+}^{\alpha-1} \rho(1)-b_{1} I_{0+}^{\alpha} \rho(1),
\end{array}\right.
$$

that is,

$$
\begin{aligned}
c_{1}= & \frac{1}{l}\left|\begin{array}{cc}
\gamma_{1}-a_{1} I_{0+}^{\alpha} \rho(0)+a_{2} I_{0+}^{\alpha-1} \rho(0) & a_{2} \\
\gamma_{2}-b_{2} I_{0+}^{\alpha-1} \rho(1)-b_{1} I_{0+}^{\alpha} \rho(1) & -\left(b_{1}+b_{2}\right)
\end{array}\right| \\
= & \frac{a_{2} b_{1}}{l} \frac{1}{\Gamma(\alpha)} \int_{0}^{1}(1-s)^{\alpha-1} \rho(s) d s \\
& +\frac{a_{2} b_{2}}{l} \frac{1}{\Gamma(\alpha-1)} \int_{0}^{1}(1-s)^{\alpha-2} \rho(s) d s-\frac{\left(b_{1}+b_{2}\right) \gamma_{1}+a_{2} \gamma_{2}}{l}, \\
c_{2}= & \frac{1}{l}\left|\begin{array}{ll}
-a_{1} & \gamma_{1}-a_{1} I_{0+}^{\alpha} \rho(0)+a_{2} I_{0+}^{\alpha-1} \rho(0) \\
-b_{1} & \gamma_{2}-b_{2} I_{0+}^{\alpha-1} \rho(1)-b_{1} I_{0+}^{\alpha} \rho(1)
\end{array}\right| \\
= & \frac{a_{1} b_{1}}{l} \frac{1}{\Gamma(\alpha)} \int_{0}^{1}(1-s)^{\alpha-1} \rho(s) d s \\
& +\frac{a_{1} b_{2}}{l} \frac{1}{\Gamma(\alpha-1)} \int_{0}^{1}(1-s)^{\alpha-2} \rho(s) d s-\frac{a_{1} \gamma_{2}-b_{1} \gamma_{1}}{l},
\end{aligned}
$$

where $l=a_{1} b_{1}+a_{1} b_{2}+a_{2} b_{1} \neq 0$. Substituting the values of $c_{1}$ and $c_{2}$ in (3.4), we get the solution given by (3.2), which completes the proof.

Remark 3.1 From the expression of the function $G(t, s)$, it is obvious that $G(t, s)$ is continuous on $I$, and is bounded. Let

$$
G^{*}=\sup \left\{\int_{0}^{1}|G(t, s)| d s: t \in I\right\} .
$$

To facilitate our discussion, let $B_{r}=\{x \in E:\|x\|<b\}, D_{r}=\left\{z \in C\left(I, E_{w}\right),\|z\| \leq r\right\}$, $\mathrm{BV}(I, \mathbb{R})$ represents the space of real bounded variation functions with its classical norm $\|\cdot\|_{\mathrm{BV}}, p, x: I \rightarrow E, f: I \times E^{3} \rightarrow E, g, h: I \times E \rightarrow E$ and $G, k_{1}, k_{2}: I \times I \rightarrow \mathbb{R}$ satisfy the following assumptions:

(1) $p$ is weakly continuous function from $I$ to $E$.

(2) For each uniformly $A C G_{*}$ function $x: I \rightarrow E$, the functions $k_{1}(t, \cdot) g(\cdot, x(\cdot))$, $k_{2}(t, \cdot) h(\cdot, x(\cdot)), f(\cdot, x(\cdot), T(x)(\cdot), S(x)(\cdot))$ are HKP integrable, $f, g, h$ are weakly-weakly continuous functions and $\int_{0}^{t} g(s, x(s)) d s, \int_{0}^{1} h(s, x(s)) d s$ are bounded on $I$. 
(3) For any $r>0$, there exist a HK-integrable function $M_{r}: I \rightarrow \mathbb{R}^{+}$and a nondecreasing continuous function $\Omega:[0, \infty) \rightarrow(0, \infty)$ such that $\|f(t, x, y, z)\| \leq M_{r}(t) \Omega(r)$ for all $t \in I,(x, y, z) \in D_{r} \times D_{r} \times D_{r}$.

(4) For each $t \in I, G(t, \cdot), k_{i}(t, \cdot) \in \mathrm{BV}(I, \mathbb{R}), i=1,2$ are continuous, i.e. the applications $t \mapsto G(t, \cdot)$ and $t \mapsto k(t, \cdot)$ are $\|\cdot\|_{\mathrm{BV}}$-continuous.

(5) The family

$$
\left\{x^{*} f(\cdot, x(\cdot), T(x)(\cdot), S(x)(\cdot)): x^{*} \in E^{*},\left\|x^{*}\right\| \leq 1\right\}
$$

is uniformly HK-integrable over $I$ for every $x \in D_{r}$.

(6) For each bounded set $X, Y, Z \subset D_{r}$, and each for each closed interval $J \subset I, t \in I$, there exists a positive constant $L_{1}, L_{2} \in(0,1)$ such that

$$
\begin{aligned}
& \beta\left(k_{1}(J, J) g(J, Y)\right) \leq L_{1} \beta(Y(J)), \quad \beta\left(k_{2}(J, J) h(J, Z)\right) \leq L_{2} \beta(Z(J)), \\
& \beta(f(t, X, Y, Z)) \leq M_{r}(t) \max \{\beta(X), \beta(Y), \beta(Z)\} .
\end{aligned}
$$

(7) There exists a constant $r_{0}>0$ such that

$$
\frac{r_{0}}{\|p\|_{\infty}+\left\|M_{r_{0}}\right\|_{\infty} \Omega\left(r_{0}\right) G^{*}}>1
$$

Now, we present the existence theorem for the problem (1.1).

Theorem 3.1 Assume that the conditions (1)-(7) and the families

$$
\begin{aligned}
& \left\{x^{*} \int_{0}^{(\cdot)} k_{1}(t, x) g\left(s, x_{n}(s)\right) d s\right\}_{n=1}^{\infty}, \quad\left\{x^{*} \int_{0}^{(\cdot)} k_{2}(t, x) h\left(s, x_{n}(s)\right) d s\right\}_{n=1}^{\infty}, \\
& \left\{x^{*} \int_{0}^{t} G(t, s) f\left(t, x_{n}(s), \int_{0}^{(\cdot)} k_{1}(t, s) g\left(s, x_{n}(s)\right), \int_{0}^{(\cdot)} k_{2}(t, s) h\left(s, x_{n}(s)\right)\right) d s\right\}_{n=1}^{\infty},
\end{aligned}
$$

are uniformly $A C G_{*}$ and equicontinuous on I for every $t \in I$ be satisfied, and let $r(K)$ be the spectral radius of the integral operator $K$ defined by

$$
(K \varphi)(t)=\int_{0}^{t} G(t, s) M_{r}(s) \varphi(s) d s, \quad \varphi \in D_{r}
$$

If $r(K)<1$, then the problem $(1.1)$ has at least one solution $x \in C\left(I, E_{w}\right)$.

Proof To simplify, we denote $m=\sup _{t \in I}\left\|k_{1}(t, \cdot)\right\|_{\mathrm{BV}}, \quad c=\sup _{t \in I}\|p(t)\|$, and $k_{0}=$ $\max \left\{\sup _{t \in I} \int_{0}^{t} g(s, x(s)) d s, \sup _{t \in I} \int_{0}^{1} h(s, x(s)) d s\right\}$. Let $c<k_{0}<\min \left(r_{0}, \frac{r_{0}}{m}\right)$. For $x \in D_{r_{0}}$ and $x^{*} \in E^{*}$ such that $\|x\|^{*} \leq 1$, we have

$$
\begin{aligned}
\left|x^{*}(T x(s))\right| & =\left|(H K) \int_{0}^{t} x^{*}\left(k_{1}(t, s) g(s, x(s))\right) d s\right| \\
& \leq\left\|x^{*}\right\| \sup _{t \in I}\left\|k_{1}(t, \cdot)\right\|_{\mathrm{BV}} \int_{0}^{1}\|g(s, x(s))\| d s \leq m \cdot k_{0} \leq r_{0},
\end{aligned}
$$


and also

$$
\sup \left\{\left|x^{*} T x\right|: x \in E^{*},\left\|x^{*}\right\| \leq 1\right\} \leq r_{0}
$$

So $T x \in D_{r_{0}}$. Similarly, we prove $S x \in D_{r_{0}}$.

Define the operator $F: C\left(I, E_{w}\right) \rightarrow C\left(I, E_{w}\right)$ by

$$
F x(t)=p(t)+\int_{0}^{1} G(t, s) f(s, x(s),(T x)(s),(S x)(s)) d s, \quad t \in I,
$$

where $G(\cdot, \cdot)$ is the Green's function defined by (3.3). Clearly the fixed points of the operator $F$ are solutions of the problem (1.1). According to the assumptions (2), (3), and (5), let $G(t, \cdot) \in \mathrm{BV}(I, \mathbb{R})$ for each $I, f(\cdot, x(\cdot), T(x)(\cdot), S(x)(\cdot))$ is HKP-integrable over $I$ for every $x \in$ $D_{r_{0}}$ and the family

$$
\left\{x^{*} f(\cdot, x(\cdot), T(x)(\cdot), S(x)(\cdot)): x^{*} \in E^{*},\left\|x^{*}\right\| \leq 1\right\}
$$

be uniformly HK-integrable over $I$ for every $x \in D_{r_{0}}$. Now, let $\mathcal{F}$ be a family of functions which are uniformly HK-integrable over an interval $I$. Then it is easy to see (the proof is in the spirit of [17], Th. 4.28) that $\mathcal{F}$ satisfies uniformly the Cauchy criterion over any closed subinterval $J \subset I$. Analogously to [17], Th. 4.27, the condition

$$
\forall \varepsilon>0 \exists \text { gauge } \gamma \text { on } I \forall P_{1}, P_{2} \gamma \text { fine-partitions } \forall f \in \mathcal{F}\left|\mathcal{S}\left(f, P_{1}\right)-\mathcal{S}\left(f, P_{2}\right)\right|<\varepsilon \text {, }
$$

implies

$$
\forall \varepsilon>0 \exists \text { gauge } \gamma \text { on } I \forall P_{1}, P_{2} \gamma \text { fine-partitions } \forall f \in \mathcal{F}\left|\mathcal{S}(f, P)-(H K) \int_{I} f(t) d t\right|<\varepsilon .
$$

Therefore, if $\mathcal{F}$ is family uniformly HK-integrable over interval $[0, b]$, then family $\mathcal{F}$ is uniformly HK-integrable over $[0, \tau]$ for every $\tau<b$. Consequently, in view of assumption (5) the family

$$
\left\{x^{*} f(\cdot, x(\cdot), T(x)(\cdot), S(x)(\cdot)): x^{*} \in E^{*},\left\|x^{*}\right\| \leq 1\right\}
$$

will be uniformly HK-integrable over any subinterval $[0, \tau] \subset I$, for every $x \in D_{r_{0}}$. This entails the weak $^{*}$-continuity of the linear functional

$$
x^{*} \in E^{*} \mapsto(H K) \int_{0}^{\tau} G(t, s) x^{*} f(s, x(s), T(x)(s), S(x)(s)) d s
$$

for all $\tau \in I$. The latter in turn means that there is $x_{t, \tau} \in E$ such that

$$
x^{*} x_{t, \tau}=(H K) \int_{0}^{\tau} x^{*} G(t, s) f(s, x(s), T(x)(s), S(x)(s)) d s, \quad \forall x^{*} \in E^{*},
$$

i.e. the function $G(t, \cdot) f(\cdot, x(\cdot), T(x)(\cdot), S(x)(\cdot))$ is HKP-integrable on $I$ and thus the operator $F$ makes sense. 
Let $r_{0}>0$, and consider the set

$$
\begin{aligned}
Q= & \left\{x \in D_{r_{0}}:\|x\|_{\infty} \leq r_{0}, \forall t_{1}, t_{2} \in I,\right. \\
& \left.\left\|x\left(t_{2}\right)-x\left(t_{1}\right)\right\| \leq\left\|p\left(t_{2}\right)-p\left(t_{1}\right)\right\|+\left\|M_{r_{0}}\right\|_{\infty} \Omega\left(r_{0}\right) \int_{0}^{1}\left|G\left(t_{2}, s\right)-G\left(t_{1}, s\right)\right| d s\right\},
\end{aligned}
$$

it is clear that the convex closed and equicontinuous subset $Q \subset D_{r_{0}} \subset C\left(I, E_{w}\right)$. We will show that $F$ satisfies the assumptions of Theorem 2.3; the proof will be given in three steps.

Step 1. We shall show that the operator $F$ maps into itself. First of all, we begin to show that $F: Q \rightarrow Q$. To see this, let $x \in Q, t \in I$. Without loss of generality, assume that $F x(t) \neq 0$. By the Hahn-Banach theorem, there exists $x^{*} \in E^{*}$ with $\left\|x^{*}\right\|=1$ and $\|F x(t)\|=\left|x^{*}(F x(t))\right|$. Thus

$$
\begin{aligned}
\|F x(t)\| & =\left|x^{*}(F x(t))\right| \leq x^{*}(p(t))+x^{*}\left(\int_{0}^{1} G(t, s) f(s, x(s),(T x)(s),(S x)(s))\right) d s \\
& \leq\|p(t)\|+\Omega\left(r_{0}\right) \sup _{t \in I} \int_{0}^{1} G(t, s) M_{r_{0}}(s) d s \\
& \leq\|p\|_{\infty}+\left\|M_{r_{0}}\right\|_{\infty} \Omega\left(r_{0}\right) G^{*} \leq r_{0},
\end{aligned}
$$

then $\|F x\|_{\infty}=\sup _{t \in I}\|F x(t)\| \leq r_{0}$. Hence $F: Q \rightarrow Q$.

Let $0<t_{1}<t_{2}$, without loss of generality, assume that $F x\left(t_{2}\right)-F x\left(t_{1}\right) \neq 0$. By the HahnBanach theorem, there exists $x^{*} \in E^{*}$ with $\left\|x^{*}\right\|=1$ and

$$
\begin{aligned}
& \left\|F x\left(t_{2}\right)-F x\left(t_{1}\right)\right\| \\
& \quad=x^{*}\left(F x\left(t_{2}\right)-F x\left(t_{1}\right)\right) \\
& \quad \leq x^{*}\left|p\left(t_{2}\right)-p\left(t_{1}\right)\right|+\int_{0}^{1}\left|G\left(t_{2}, s\right)-G\left(t_{1}, s\right)\right| \cdot\left|x^{*}(f(s, x(s),(T x)(s),(S x)(s)))\right| d s \\
& \quad \leq x^{*}\left|p\left(t_{2}\right)-p\left(t_{1}\right)\right|+\left\|M_{r_{0}}\right\|_{\infty} \Omega\left(r_{0}\right) \int_{0}^{1}\left|G\left(t_{2}, s\right)-G\left(t_{1}, s\right)\right| d s \\
& \quad \leq\left\|p\left(t_{2}\right)-p\left(t_{1}\right)\right\|+\left\|M_{r_{0}}\right\|_{\infty} \Omega\left(r_{0}\right) \int_{0}^{1}\left|G\left(t_{2}, s\right)-G\left(t_{1}, s\right)\right| d s .
\end{aligned}
$$

This estimation shows that $F$ maps $Q$ into itself.

Step 2. We will show that the operator $F$ is weakly sequentially continuous. To see this, by Lemma 9 of [18], a sequence $x_{n}(\cdot)$ weakly convergent to $x(\cdot) \in Q$ if and only if $x_{n}(\cdot)$ tends weakly to $x(t)$ for each $t \in I$. Because $g(s, \cdot)$ is weakly-weakly sequentially continuous, so if $x_{n} \rightarrow x$ in $C\left(I, E_{w}\right)$, then $g\left(s, x_{n}(s)\right) \rightarrow g(s, x(x))$ and $h\left(s, x_{n}(s)\right) \rightarrow h(s, x(x))$ in $C\left(I, E_{w}\right)$, and by Theorem 2.2 (see our assumptions (3.5), (3.6)), we have

$$
\lim _{n \rightarrow \infty} \int_{0}^{1} k_{1}(t, s) g\left(s, x_{n}(s)\right) d s=\int_{0}^{1} k_{1}(t, s) g(s, x(s)) d s
$$

weakly in $E$ for each $t \in I$ and $T x_{n}(t) \rightarrow T x(t)$.

Similarly, we have

$$
\lim _{n \rightarrow \infty} \int_{0}^{1} k_{2}(t, s) h\left(s, x_{n}(s)\right) d s=\int_{0}^{1} k_{2}(t, s) h(s, x(s)) d s
$$


weakly in $E$ for each $t \in I$ and $S x_{n}(t) \rightarrow S x(t)$. Therefore, the operator $T, S$ are weakly sequentially continuous in $Q$.

Moreover, because $f$ is weakly-weakly sequentially continuous,

$$
f\left(s, x_{n}(s),\left(T x_{n}\right)(s),\left(S x_{n}\right)(s)\right) \rightarrow f(s, x(s),(T x)(s),(S x)(s))
$$

weakly in $E$, for each $I$. Now, applying assumption (5), Theorem 5 in [19] and Lemma 25 in [20], then the function $G(t, \cdot) a(\cdot) f\left(\cdot, x_{n}(\cdot),\left(T x_{n}\right)(\cdot),\left(S x_{n}\right)(\cdot)\right)$ is HKP-integrable on $I$ for every $n \geq 1$, by Theorem 2.2 and assumption (3.6), we have

$$
\begin{gathered}
\lim _{n \rightarrow \infty} \int_{0}^{1} G(t, s) a(s) f\left(s, x_{n}(s),\left(T x_{n}\right)(s),\left(S x_{n}\right)(s)\right) d s \\
=\int_{0}^{1} G(t, s) a(s) f(s, x(s),(T x)(s),(S x)(s)) d s,
\end{gathered}
$$

then $F\left(x_{n}\right) \rightarrow F(x)$ in $C\left(I, E_{w}\right)$.

Step 3. We show that the operator $F: Q \rightarrow Q$ is power-convex condensing.

Let $B=\overline{\mathrm{co}} F(Q) \subset Q$. Obviously, $B$ is bounded, convex, and closed, and $F(\overline{\mathrm{c}} F(Q)) \subset$ $F(B) \subset \overline{\mathrm{co}} F(Q)$, i.e., $F: B \rightarrow B$. By Lemma $2.1, B$ is equicontinuous in $C\left(I, E_{w}\right)$. Obviously, $F$ is bounded and continuous. Set $x_{0} \in F$, we will prove that there exists $n_{0}$, such that, for any bounded $V \subset B$,

$$
\beta\left(F^{\left(n_{0}, x_{0}\right)}(V)\right) \leq \beta(V)
$$

By $V \subset B \subset Q, F(V)$ is equicontinuous. Then $F^{\left(2, x_{0}\right)}(V)$ is equicontinuous from $F^{\left(2, x_{0}\right)}(V)=$ $F\left(\overline{\mathrm{co}} F(V), x_{0}\right) \subset F(Q)$. Generally, $\forall n \in N, F^{\left(n, x_{0}\right)}(V)$ is equicontinuous. Since $F^{\left(n, x_{0}\right)}(V)$ is bounded, By Lemma 2.2,

$$
\beta\left(F^{\left(n, x_{0}\right)}(V)\right)=\max _{t \in I}\left(F^{\left(n, x_{0}\right)}(V)(t)\right), \quad n=2,3, \ldots
$$

Now fix $t \in I$ and divide the interval $I$ into $n$ parts $0=t_{0}<t_{1}<\cdots<t_{n}=1$, for $s_{1}, s_{2}, s_{3}, r_{1}, r_{2}, r_{3} \in T_{i}=\left[t_{i-1}, t_{i}\right]$ and $\epsilon>0$, there exists $\delta>0$ such that

$$
\left|M_{r}\left(s_{1}\right) G\left(t, s_{2}\right) v\left(s_{3}\right)-M_{r}\left(r_{1}\right) G\left(t, r_{2}\right) v\left(r_{3}\right)\right|<\epsilon,
$$

if $\left|s_{1}-r_{1}\right|<\delta,\left|s_{2}-r_{2}\right|<\delta,\left|s_{2}-r_{2}\right|<\delta$.

Let $\gamma_{i}=\sup _{s \in T_{i}}|G(t, s)|=\left|G\left(t, s_{i}\right)\right|,\left|M_{r}\left(\tau_{i}\right)\right|=\sup _{s \in T_{i}}\left|M_{r}(s)\right|, s_{i}, \tau_{i} \in T_{i}$ and $V_{i}=\{x(s): x \in$ $\left.V, s \in T_{i}\right\}$. By the Ambrosetti lemma there exists $q_{i} \in T_{i}$ such that $\beta\left(V_{i}\right)=v\left(q_{i}\right)$, then

$$
\begin{aligned}
\beta\left(F^{1, x_{0}}(V)(t)\right) & =\beta(F(V)(t)) \\
& =\beta\left(p(t)+\int_{0}^{1} G(t, s) f(s, V(s),(T V)(s),(S V)(s)) d s\right) \\
& =\beta\left(\int_{0}^{1} G(t, s) f(s, V(s),(T V)(s),(S V)(s)) d s\right) .
\end{aligned}
$$


By Theorem 2.1, we obtain

$$
\begin{aligned}
& \int_{0}^{1} G(t, s) f(s, V(s),(T V)(s),(S V)(s)) d s \\
& \quad=\sum_{i=1}^{n} \int_{T_{i}} G(t, s) f(s, V(s),(T V)(s),(S V)(s)) d s \\
& \quad \in \sum_{i=1}^{n} \mu\left(T_{i}\right) \overline{\operatorname{co}}\left\{G(t, s) f(s, x(s),(T x)(s),(S x)(s)): s \in T_{i}, x \in V\right\} \\
& \quad \subset \sum_{i=1}^{n} \mu\left(T_{i}\right) \overline{\operatorname{co}}\left(\bigcup_{|\gamma| \leq \gamma_{i}} \gamma f\left(T_{i} \times V\left(T_{i}\right) \times T(V)\left(T_{i}\right) \times S(V)\left(T_{i}\right)\right)\right) .
\end{aligned}
$$

Furthermore, by the properties of $\beta$, we have

$$
\begin{aligned}
& \beta\left(\int_{0}^{1} G(t, s) f(s, V(s), T(V)(s), S(V)(s)) d s\right) \\
& \quad \leq \beta\left(\sum_{i=1}^{n} \mu\left(T_{i}\right) \overline{c o}\left\{G(t, s) f(s, x(s),(T x)(s),(S x)(s)): s \in T_{i}, x \in V\right\}\right) \\
& \quad \leq \sum_{i=1}^{n} \mu\left(T_{i}\right) \beta\left(\bigcup_{|\gamma| \leq \gamma_{i}} \gamma f\left(T_{i} \times V\left(T_{i}\right) \times T(V)\left(T_{i}\right) \times S(V)\left(T_{i}\right)\right)\right) \\
& \quad=\sum_{i=1}^{n} \mu\left(T_{i}\right) \sup _{s \in T_{i}}|G(t, s)| \beta\left(f\left(T_{i} \times V\left(T_{i}\right) \times T(V)\left(T_{i}\right) \times S(V)\left(T_{i}\right)\right)\right) \\
& \quad \leq \sum_{i=1}^{n} \mu\left(T_{i}\right)\left|G\left(t, s_{i}\right)\right| \sup _{s \in T_{i}} M_{r}(s) \max \left\{\beta\left(V\left(T_{i}\right)\right), \beta\left(T(V)\left(T_{i}\right)\right), \beta\left(S(V)\left(T_{i}\right)\right)\right\} \\
& \quad \leq \sum_{i=1}^{n} \mu\left(T_{i}\right) \gamma_{i} M_{r}\left(\tau_{i}\right) \max _{i}\left\{\beta\left(V\left(T_{i}\right)\right), L_{1} \beta\left(V\left(T_{i}\right)\right), L_{2} \beta\left(V\left(T_{i}\right)\right)\right\} \\
& \quad \leq \sum_{i=1}^{n} \mu\left(T_{i}\right) \gamma_{i} M_{r}\left(\tau_{i}\right) \max \left\{1, L_{1}, L_{2}\right\} \cdot \beta\left(V\left(T_{i}\right)\right) \\
& \quad \leq \sum_{i=1}^{n} \mu\left(T_{i}\right) \gamma_{i} M_{r}\left(\tau_{i}\right) \beta\left(V\left(T_{i}\right)\right) .
\end{aligned}
$$

So

$$
\begin{aligned}
\beta\left(\int_{0}^{1} G(t, s) f(s, V(s), T(V)(s), S(V)(s)) d s\right) & \leq\left|\sum_{i=1}^{n} \mu\left(T_{i}\right) \gamma_{i} \sup _{s \in T_{i}} M_{r}(s) \beta\left(V\left(T_{i}\right)\right)\right| \\
& \leq \sum_{i=1}^{n} \mu\left(T_{i}\right)\left|G\left(t, s_{i}\right)\right| M_{r}\left(\tau_{i}\right) v\left(q_{i}\right),
\end{aligned}
$$

where $s_{i}, \tau_{i}, q_{i} \in T_{i}$, and

$$
\left|M_{r}(s) G(t, s) v(s)-M_{r}\left(\tau_{i}\right) G\left(t, s_{i}\right) v\left(q_{i}\right)\right|<\epsilon, \quad \text { for } s \in T_{i}
$$


we have

$$
M_{r}\left(\tau_{i}\right) G\left(t, s_{i}\right) v\left(q_{i}\right) \mu\left(T_{i}\right) \leq \int_{T_{i}} M_{r}(s) G(t, s) v(s) d s+\epsilon \mu\left(T_{i}\right) .
$$

Thus

$$
\begin{gathered}
\beta\left(\int_{0}^{1} G(t, s) f(s, V(s), T(V)(s), S(V)(s)) d s\right) \\
\leq \int_{0}^{1} G(t, s) M_{r}(s) v(s) d s+\epsilon \sum_{i=1}^{n} \mu\left(T_{i}\right) .
\end{gathered}
$$

Because $\epsilon$ is arbitrarily small, we get

$$
\begin{aligned}
& \beta\left(\left\{p(t)+\int_{0}^{1} G(t, s) f(s, x(s),(T x)(s),(S x)(s)) d s: x \in V\right\}\right) \\
& \quad \leq \beta(V) \int_{0}^{1} G(t, s) M_{r}(s) d s,
\end{aligned}
$$

i.e.

$$
\beta\left(F^{\left(1, x_{0}\right)}(V)(t)\right) \leq \beta(V) \int_{0}^{1} G(t, s) M_{r}(s) d s=\beta(V) \cdot K \varphi_{0}(t),
$$

where $\varphi_{0}(t) \equiv 1, \forall t \in I$.

By the equicontinuity of $F^{\left(1, x_{0}\right)}(V)=F(V)$ and $G(t, s) f\left(s,\left(\overline{\operatorname{co}}\left\{F^{\left(1, x_{0}\right)}(V)(s), x_{0}\right\}\right)\right.$, $\left.\left(T \overline{\operatorname{co}}\left\{F^{\left(1, x_{0}\right)}(V)(s), x_{0}\right\}\right),\left(S \overline{\operatorname{co}}\left\{F^{\left(1, x_{0}\right)}(V)(s), x_{0}\right\}\right)\right)$ is equicontinuous. Therefore,

$$
\begin{aligned}
\beta( & \left.\left(F^{\left(2, x_{0}\right)}(V)\right)(t)\right) \\
= & \beta\left(F \overline{\mathrm{co}}\left\{\left(F^{\left(1, x_{0}\right)}(V)\right)(t), x_{0}\right\}\right) \\
= & \beta\left(\int _ { 0 } ^ { 1 } G ( t , s ) f \left(s,\left(\overline{\mathrm{co}}\left\{F^{\left(1, x_{0}\right)}(V)(s), x_{0}\right\}\right),\left(T \overline{\operatorname{co}}\left\{F^{\left(1, x_{0}\right)}(V)(s), x_{0}\right\}\right),\right.\right. \\
& \left.\left.\left(S \overline{\operatorname{co}}\left\{F^{\left(1, x_{0}\right)}(V)(s), x_{0}\right\}\right)\right) d s\right) \\
\leq & \int_{0}^{1} \beta\left(\left(G ( t , s ) f \left(s,\left(\overline{\operatorname{co}}\left\{F^{\left(1, x_{0}\right)}(V)(s), x_{0}\right\}\right),\left(T \overline{\operatorname{co}}\left\{F^{\left(1, x_{0}\right)}(V)(s), x_{0}\right\}\right),\right.\right.\right. \\
& \left.\left.\left(S \overline{\operatorname{co}}\left\{F^{\left(1, x_{0}\right)}(V)(s), x_{0}\right\}\right)\right)\right) d s \\
\leq & \int_{0}^{1} G(t, s) M_{r}(s) \beta\left(\overline{\operatorname{co}}\left\{F^{\left(1, x_{0}\right)}(V)(s), x_{0}\right\}\right) d s \\
= & \int_{0}^{1} G(t, s) M_{r}(s) \beta\left(F^{\left(1, x_{0}\right)}(V)(s)\right) d s \\
\leq & \beta(V) K^{2} \varphi_{0}(t) .
\end{aligned}
$$

Generally,

$$
\beta\left(\left(F^{\left(n, x_{0}\right)}(V)\right)(t)\right) \leq \beta(V) K^{n} \varphi_{0}(t) .
$$


Since $r(K)<1$, let $\varepsilon=\frac{1-r(K)}{2}$, then $\exists m_{0}>0$, when $n>m_{0}$,

$$
\begin{aligned}
\max _{t \in I}\left|K^{n} \varphi_{0}(t)\right| & =\left\|K^{n} \varphi_{0}\right\| \leq\left\|K^{n}\right\|\left\|\varphi_{0}\right\|=\left\|K^{n}\right\| \\
& \leq(r(K)+\varepsilon)^{n}=\left(\frac{1+r(K)}{2}\right)^{n}<1 .
\end{aligned}
$$

Set $n_{0}>m_{0}$, then $\forall t \in I$,

$$
\begin{aligned}
\beta\left(\left(F^{\left(n, x_{0}\right)}(V)\right)(t)\right) & \leq \beta(V) \cdot K^{n_{0}} \varphi_{0}(t) \leq\left\|K^{n_{0}} \varphi_{0}\right\| \beta(V) \\
& \leq\left(\frac{1+r(K)}{2}\right)^{n_{0}} \beta(V) \leq \beta(V) .
\end{aligned}
$$

By (3.7), $\beta\left(F^{\left(n, x_{0}\right)}(V)\right) \leq \beta(V)$. Therefore, $F: V \rightarrow V$ is convex-power condensing. By Theorem 2.3, $F$ has one fixed point in $C\left(I, E_{w}\right)$, i.e., the problem (1) has at least one solution in $C\left(I, E_{w}\right)$.

Remark 3.2 The assumption (3) should instead agree with the following condition: The function $f$ is weakly-weakly sequentially continuous (for each convergent sequence $\left.\left\{t_{n}\right\} \subset[0,1]\right)$ and for all weakly convergent sequences $\left\{x_{n}\right\},\left\{y_{n}\right\},\left\{z_{n}\right\} \subset E$, the sequence $\left\{f\left(t_{n}, x_{n}, y_{n}, z_{n}\right)\right\}$ is weakly convergent in $\left.E\right)$ such that, for all $r>0$ and $\varepsilon>0$, there exists $\delta_{\varepsilon, r}>0$ such that

$$
\left\|\int_{\tau}^{t} f(s, x(s),(T x)(s),(S x)(s)) d s\right\|<\varepsilon, \quad \forall|t-\tau|<\delta_{\varepsilon, r}, \forall x \in D_{r} .
$$

Proof Let $r>0$ and $x^{*} \in E^{*}$ such that $\left\|x^{*}\right\| \leq 1$. For $0 \leq t_{1}<t_{2} \leq 1$, we have

$$
\left|x^{*} \int_{t_{1}}^{t_{2}} f(s, x(s),(T x)(s),(S x)(s)) d s\right| \leq(H K) \int_{t_{1}}^{t_{2}} x^{*} f(s, x(s)),(T x)(s),(S x)(s) d s
$$

Because $s \mapsto M_{r}(s)$ is Henstock-Kurzweil integrable and $\left|x^{*} f(s, x(s)),(T x)(s),(S x)(s)\right| \leq$ $\left\|x^{*}\right\|\|f(s, x(s),(T x)(s),(S x)(s))\| \leq M_{r}$ for all $s \in I$, then by [21], Corollary 4.62, $s \mapsto$ $x^{*} f(s, x(s),(T x)(s),(S x)(s))$ is absolutely Henstock-Kurzweil integrable on $\left[t_{1}, t_{2}\right] \subset I$ and

$$
\left|(H K) \int_{t_{1}}^{t_{2}} x^{*} f(s, x(s),(T x)(s),(S x)(s)) d s\right| \leq(H K) \int_{t_{1}}^{t_{2}} M_{r}(s) d s
$$

Thus

$$
\begin{aligned}
\left\|\int_{t_{1}}^{t_{2}} f(s, x(s),(T x)(s),(S x)(s)) d s\right\| & =\sup _{\left\|x^{*}\right\| \leq 1}\left|x^{*} \int_{t_{1}}^{t_{2}} f(s, x(s), T x(s), S x(s)) d s\right| \\
& \leq(H K) \int_{t_{1}}^{t_{2}} M_{r}(s) d s .
\end{aligned}
$$

Due to the continuity of the primitive function of the Henstock-Kurzweil integral, we have a $t_{2}$ less than $\varepsilon$ and sufficiently close to $t_{1}$, and the proof is completed. 


\section{Conclusions}

In this paper, we use the techniques of the measure of weak noncompactness and Henstock-Kurzweil-Pettis integrals to discuss the existence theorem of weak solutions for a class of nonlinear fractional integrodifferential equations in a nonreflexive Banach space equipped with the weak topology.

\section{Competing interests}

The authors declare that they have no competing interests.

\section{Authors' contributions}

All authors contributed equally to the writing of this paper. All authors read and approved the final manuscript.

\section{Acknowledgements}

The authors wish to thank the referees for their endeavors and valuable comments. This work is supported by National Natural Science Foundation of China (11061031).

Received: 10 August 2016 Accepted: 11 November 2016 Published online: 25 November 2016

\section{References}

1. Agarwal, RP, Benchohra, M, Hamani, S: A survey on existence result for boundary value problem of nonlinear fractional differential equations and inclusions. Acta Appl. Math. 109, 973-1033 (2010)

2. Benchohra, M, Graef, JR, Hamani, S: Existence results of nonlinear fractional differential equations on reflexive Banach spaces. Electron. J. Qual. Theory Differ. Equ. 54, 1-10 (2010)

3. Agarwal, R, O'Regan, D, Sikorska Nowak, A: The set of solutions of integrodifferential equations and the Henstock-Kurzweil-Pettis integral in Banach spaces. Bull. Aust. Math. Soc. 78, 507-522 (2008)

4. Cichoń, M: Weak solutions of differential equations in Banach spaces. Discuss. Math., Differ. Incl. 15, 5-14 (1995)

5. Benchohra, M, Hamani, S, Ntouyas, SK: Boundary value problem for differential equations with frcational order and nonlocal conditions. Nonlinear Anal. TMA 71, 2391-2396 (2009)

6. Kubiaczyk, I, Szufla, S: Kenser's theorem for weak solutions of ordinary differential equations in Banach spaces. Publ. Inst. Math. 32, 99-103 (1982)

7. Kubiaczyk, I: On the existence of solutions of differential equations in Banach spaces. Bull. Pol. Acad. Sci., Math. 33, 607-614 (1985)

8. O'Regan, D: Operator equations in Banach space relative to the weak topology. Arch. Math. (Basel) 71, 123-136 (1998)

9. Zhou, WX, Chu, YD: Existence of solutions for fractional differential equations with multi-point boundary conditions. Commun. Nonlinear Sci. Numer. Simul. 17, 1142-1148 (2012)

10. Bouffak, M, Mostefai, FZ: Weak solutions for nonlinear fractional differential equations with integral boundary conditions in Banach spaces. Opusc. Math. 32, 31-39 (2012)

11. Li, BL, Gou, HD: On the existence of weak solutions of nonlinear integral equations in Banach spaces. Mediterr. J. Math. 13(5), 2633-2643 (2016)

12. DeBlasi, FS: On a property of the unit sphere in Banach space. Bull. Math. Soc. Sci. Math. Roum. 21, $259-262$ (1977)

13. Cichoń, M, Kubiaczyk, I, Sikorska Nowak, A: The Henstock-Kurwzeil-Pettis integrals and existence theorems for the Cauchy problem. Czechoslov. Math. J. 54, 279-289 (2004)

14. Cichoń, M: Convergence theorem for the Henstock-Kurwzeil-Pettis integrals. Acta Math. Hung. 92, 75-82 (2001)

15. Hussain, N, Taoudi, MA: Krasnoselskii-type fixed point theorems with applications to Volterra integral equations. Fixed Point Theory Appl. 2013, 196 (2013)

16. Pettis, BJ: On integration in vector space. Trans. Am. Math. Soc. 44, 277-304 (1938)

17. Kurtz, D, Swartz, C: Theories of Integration: The Integrals of Riemann, Lebesque, Henstock-Kurzweil and McShane. World Scientific, Singapore (2004)

18. Mitchell, AR, Smith, C: An existence theorem for weak solutions of differential equations in Banach spaces. In: Nonlinear Equations in Abstract Spaces, Orlando, pp. 387-404 (1978)

19. Di Piazza, L: Kurzweil-Henstock type integration on Banach spaces. Real Anal. Exch. 29(2), $543-555$ (2003)

20. Satco, B: A Kolmós-type theorem for the set-valued Henstock-Kurzweil-Pettis integrals and applications. Czechoslov. Math. J. 56(131), 1029-1047 (2006)

21. Gordon, RA: The Integrals of Lebesgue, Denjoy, Perron and Henstock. Am. Math. Soc., Providence (1994) 